\title{
Spindle positioning in the stem cell niche
}

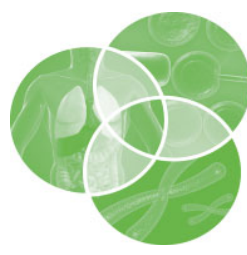

Swathi Yadlapalli* and Yukiko M. Yamashita*

Stem cells are the source of differentiated cells that constitute tissues and organs. Two fundamental characteristics of stem cells are their abilities to self-renew stem cell identity and to produce differentiated cells, the balance of which can be achieved by asymmetric stem cell division. Many stem cells have been shown to reside in a stem cell niche, the home of stem cells that regulates the stem cell behavior. Recent studies have revealed the critical contribution of cytoskeletons in achieving asymmetric stem cell division: mitotic spindles in dividing stem cells are often oriented with respect to the stem cell niche, which is supported by concerted actions of microtubule networks and components at the cell membrane such as adherens junctions, the actin cytoskeleton, and the extracellular matrix. In this article, we review the mechanism of stem cell spindle orientation, with emphasis on its relationship with the stem cell niche, and discuss how it contributes to tissue development and homeostasis. ๑ 2011 Wiley Periodicals, Inc.

How to cite this article:

WIREs Dev Biol 2012, 1:215-230. doi: 10.1002/wdev.16

\section{INTRODUCTION}

S tem cells are essential contributors to tissue development, homeostasis, and repair. Throughout the life of an organism, stem cells are required to proliferate and supply differentiated but short-lived cells such as blood, skin, intestinal epithelium, and sperm cells. The main function of adult stem cells is to generate identical copies of themselves (self-renewal) as well as to give rise to various differentiated cells that comprise mature tissue. Many stem cells studied to date, in particular those from invertebrate model systems, achieve this function through asymmetric cell division-i.e., one of the daughter cells adopts the fate of its mother, whereas the other adopts a more committed fate, initiating differentiation. ${ }^{1}$ Asymmetric cell division is a critical mechanism to maintain tissue homeostasis, preventing both overproliferation of stem cells, which can lead to tumor formation, and depletion of the stem cell pool, which can lead to tissue aging/degeneration. ${ }^{1,2}$

Studies in several model systems have suggested two distinct mechanisms by which asymmetric cell

*Correspondence to: yukikomy@umich.edu;swathi@umich.edu Department of Cell and Developmental Biology, Life Sciences Institute, Center for Stem Cell Biology, University of Michigan, Ann Arbor, MI, USA division can be achieved.1,3 The first mechanism involves extrinsic fate determinants provided by the stem cell niche, the microenvironment that instructs stem cell identity, and relies on the asymmetric placement of daughter cells into the inside versus outside of a stem cell niche (Figure 1(a)). Daughter cells of a stem cell division may initially have equivalent developmental potential, but they may acquire different fates owing to exposure to different external signals. In many model systems studied to date such as Drosophila germline stem cells (GSCs) as described below, stem cells divide asymmetrically by orienting their mitotic spindle perpendicular to the niche surface so that only one daughter cell maintains contact with the niche and retains stem cell identity, whereas the other daughter displaced away from the niche begins a program of differentiation. ${ }^{4}$ Alternatively, the second mechanism involves intrinsic fate determinants and relies on the asymmetric partitioning of fate determinants to the daughter cells ${ }^{5}$ (Figure 1(b)). Cells use apical-basal or planar polarity of the surrounding tissue to set up an axis along which to polarize the distribution of fate determinants and to orient the mitotic spindle so that these determinants are inherited by only one daughter cell upon cell division. The fate determinants, either extrinsic or intrinsic, ultimately influence nucleus, leading to 
FIGURE 1 | Mechanisms of asymmetric stem cell division. (a) Asymmetric stem cell division by extrinsic fate determinants provided by the stem cell niche. Stem cells divide asymmetrically by orienting the mitotic spindle such that only one daughter remains in the stem cell niche and has access to signals necessary for maintaining stem cell identity. Here, daughter cells that initially have equivalent developmental potential acquire different fates by placement in distinct cellular microenvironments. (b) Asymmetric stem cell division by intrinsic fate determinants. Cell fate determinants are unequally partitioned into the daughter cells, thus leading to daughters with distinct fates. Shown here are the self-renewing (red) factors inherited by the stem cell/or and differentiation (blue) factors inherited by the differentiating daughter cell. (a) Extrinsic regulation

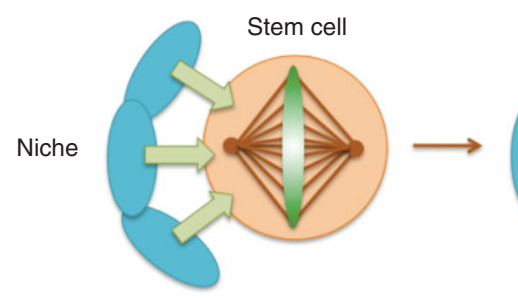

(b)

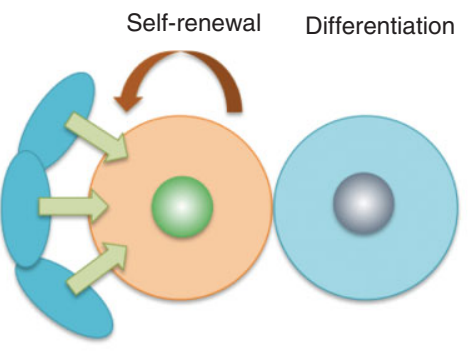

Intrinsic regulation

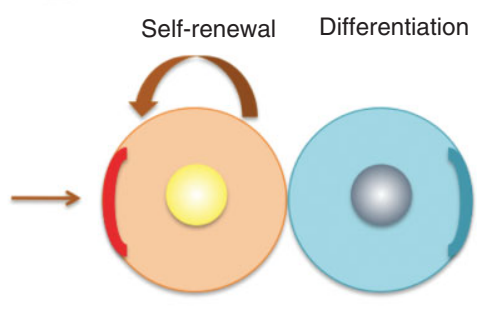

Cell polarity (apical-basal

or planar) distinct expression profiles responsible for cell fates (Figure 1(a) and (b)). For example, in Drosophila neuroblast, Prospero, which is inherited into the differentiating daughter [ganglion mother cell (GMC)] as an asymmetrically segregating cytoplasmic/cortical factor, translocates into nucleus and promotes differentiation. ${ }^{6-8}$ It is also possible that asymmetry in the nucleus (such as asymmetry in epigenetic state of two daughter cells) contributes to asymmetric stem cell division. Although epigenetic changes clearly play fundamental roles in regulation of cell fate during differentiation, it is currently unknown whether and how distinct epigenetic states are implemented at the time of asymmetric stem cell division. Stem cells are also known to divide symmetrically by orienting the spindle such that the niche contact or intrinsic fate determinants are equally segregated into two daughter cells. ${ }^{1}$ This generates two stem cells to increase stem cell number during development or to compensate for stem cell loss following injury. In general, cells use two different strategies to set up the orientation of cleavage plane in response to intrinsic or extrinsic cues. Stem cells may be oriented/polarized throughout the cell cycle-in other words, proper spindle orientation is predetermined before cells enter mitosis. ${ }^{4}$ Alternatively, cells can use programed spindle rotation during mitosis to acquire the desired alignment. ${ }^{9,10}$ The latter strategy might be especially advantageous in cases in which cells switch between symmetric versus asymmetric divisions.

In this review, we summarize recent advances in spindle orientation and asymmetric stem cell division in the context of the stem cell niche and attempt to draw an emerging picture of niche-dependent asymmetric stem cell division and its implication in tissue development and homeostasis.

\section{DROSOPHILA MALE GSCs AND SOMATIC CYSCs}

\section{Architecture of the GSC and CySC Niche}

The Drosophila male and female germlines are among the best understood model systems for the regulation of stem cell behavior and asymmetric stem cell division controlled by the stem cell niche. Drosophila male GSCs reside in a niche that instructs their self-renewal. At the apical tip of the testis, approximately 8-10 GSCs lie in a rosette around a cluster of post-mitotic support cells called hub cells, a major component of the GSC niche. ${ }^{11}$ Drosophila male GSCs always divide asymmetrically by keeping one daughter attached to the hub and displacing the other away from the hub, yielding a self-renewing GSC and a differentiating cell, called a gonialblast (GB), respectively (Figure 2(a)). GBs further undergo four mitotic divisions with incomplete cytokinesis, producing clusters of 16 interconnected spermatogonia, which produce spermatocytes that then commit to meiosis and ultimately differentiate into sperms. The testicular niche also contains a second stem cell type called cyst stem cells (CySCs; also known as cyst progenitor cells). The function of CySCs, together with the hub cells, is to create a niche for GSCs ${ }^{12}$ (Figure 2(a)). A pair of CySCs encapsulates a GSC and provides essential signals for GSC identity. ${ }^{13,14}$ Similar to GSCs, CySCs divide asymmetrically to generate a continuous supply of non-mitotic somatic support cells called cyst cells, which encapsulate and escort differentiating germ cells. ${ }^{15}$ A pair of 
(a) Drosophila testis

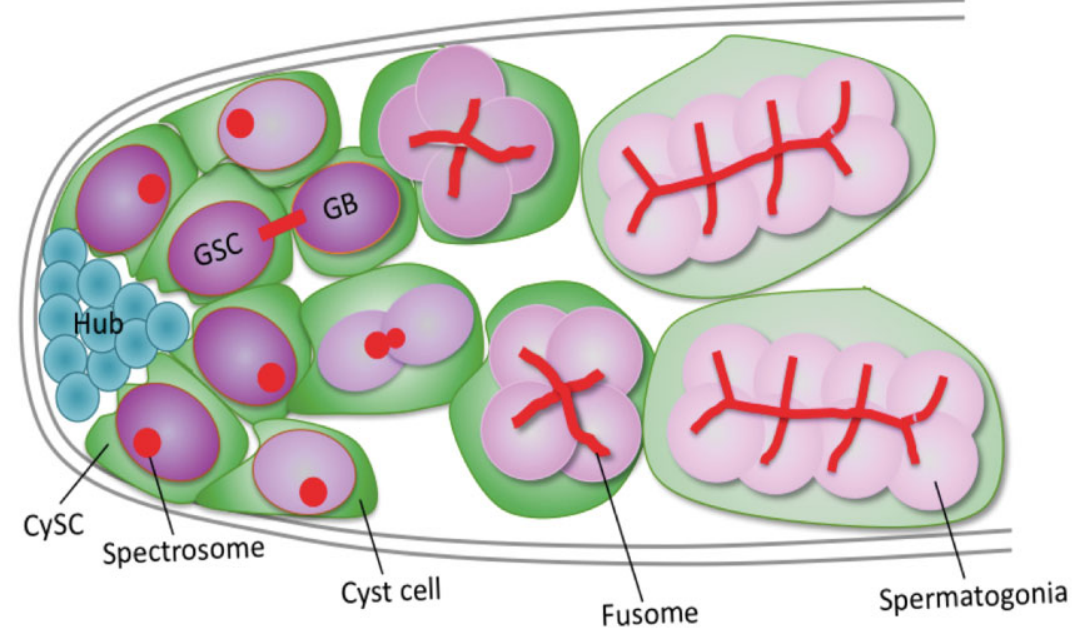

(c) Drosophila ovariole

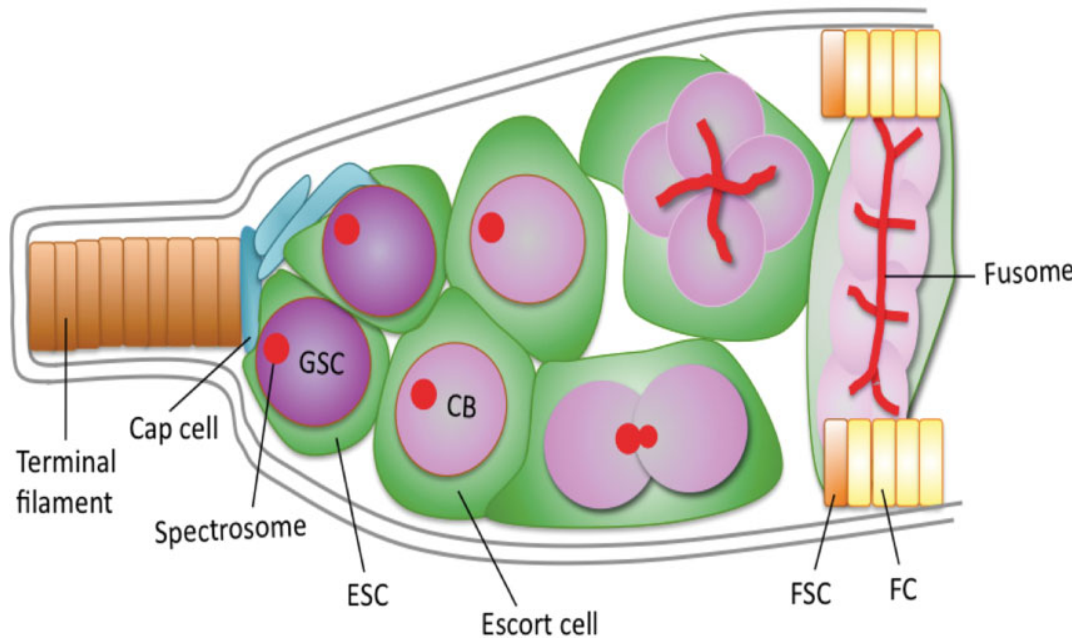

(b) JAK-STAT signaling

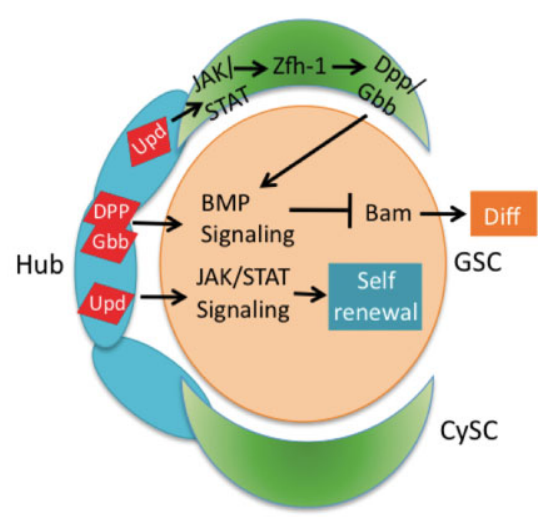

(d)

DPP/GBB signaling

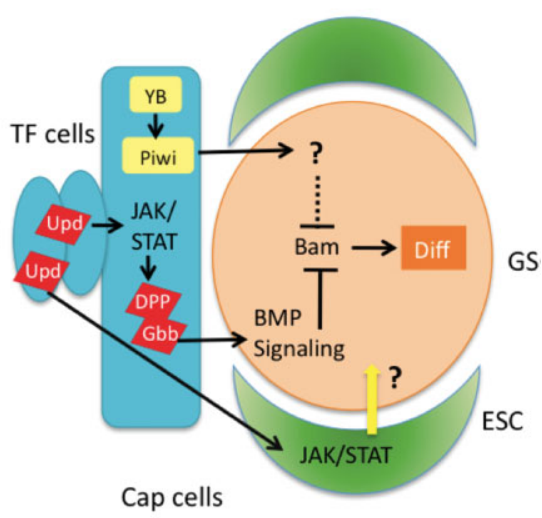

FIGURE 2 | Drosophila male and female germline and somatic stem cells. (a) At the apical tip of the testis, germline stem cells (GSCs), and cyst stem cells (CySCs) are physically attached to the hub cells via an E-cadherin-based adherens junction. GSCs divide asymmetrically where one of the daughters maintains stem cell identity and the other initiates differentiation as a gonialblast (GB) (see also Figure 3). GBs further undergo four synchronous divisions with incomplete cytokinesis, producing clusters of 16 interconnected spermatogonia, which give rise to spermatocytes and ultimately to sperm. A pair of CySCs encapsulates a GSC and provides essential signals for GSC identity. CySCs divide asymmetrically to self-renew and produce somatic support cells called cyst cells. A pair of cyst cells envelop each GB and its progeny, providing signals mediating differentiation. (b) At the anterior tip of the ovariole, GSCs are attached to the cap cells via adherens junctions. GSCs divide asymmetrically in order to self-renew as well as to produce a cystoblast (CB) that initiates differentiation (see also Figure 3). CB further divides four times to give rise to 16 germ cell cysts interconnected by the fusome, of which only one ultimately becomes an oocyte while the remaining 15 serve as nurse cells. Escort stem cells (ESCs) encapsulate the GSC, while their daughters, escort cells, encapsulate the differentiating germ cells. (c) Hub cells express unpaired (Upd), which activates STAT in GSCs and CySCs required for stem cell self-renewal. Male GSCs also require Dpp and Gbb expressed in hub cells and CySCs for self-renewal. (d) Female GSCs require BMP and Piwi/YB signaling from cap cells for self-renewal. In addition, GSC self-renewal also requires Janus kinase-signal transducer and activator of transcription (JAK-STAT) activation in the ESCs, which mediates unknown signaling to GSCs.

cyst cells envelops each GB and its progeny, providing signals that mediate differentiation. ${ }^{16}$ GSCs and CySCs are physically attached to the hub cells by adherens junctions consisting of Drosophila epithelial
(DE)-cadherin and $\beta$-catenin/Armadillo, which are concentrated at the cell cortex adjacent to the hub ${ }^{4}$ (Figure 3(a) and (b)). Indeed, cell adhesion between GSCs and hub cells, as well as between CySCs and 
(a) Drosophila male GSCs

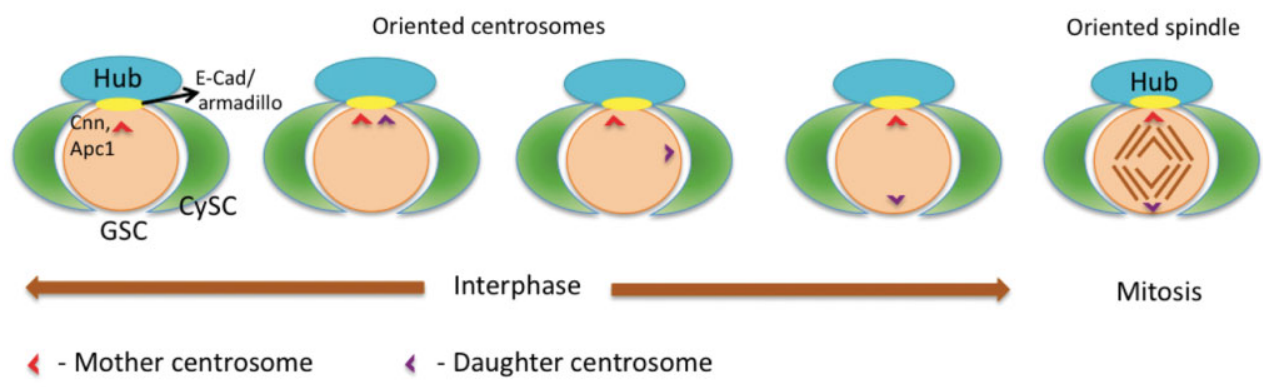

(b) Drosophila male CySCs

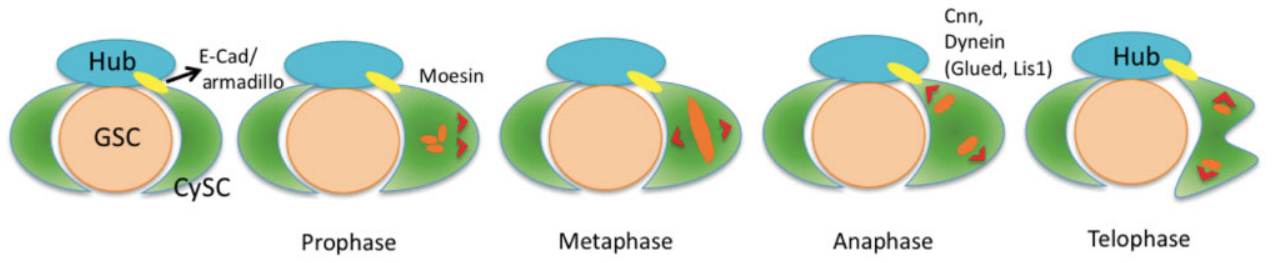

(c) Drosophila female GSCs

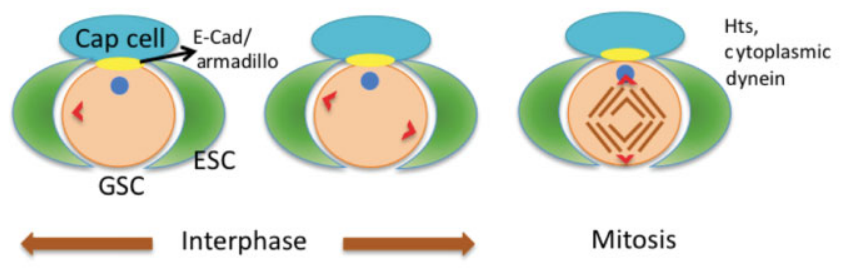

- Spectrosome

FIG URE 3 | Asymmetric cell division in Drosophila male and female germline and somatic stem cells. (a-c) Male and female germline and somatic stem cells attach to the hub cells by adherens junctions consisting of Drosophila epithelial (DE)-cadherin and Armadillo. (a) Male germline stem cells (GSCs) always orient their spindle perpendicular to the hub. This stereotypical orientation of mitotic spindle is prepared by the precisely controlled positioning of the centrosomes during interphase. Specifically, the mother centrosome (red) normally remains adjacent to the hub and is inherited by the GSC, where as the daughter centrosome (purple) migrates to the opposite side of the cell and is inherited by the gonialblast (GB). Male GSCs require centrosomin ( $\mathrm{Cnn}$ ) and ademomatous polyposis coli 2 (Apc2) proteins to correctly orient the centrosomes during interphase. (b) In contrast to GSCs, cyst stem cells (CySCs) divide asymmetrically by repositioning their spindle during anaphase. The spindle poles rock back and forth until the onset of anaphase, and then one of the poles moves closer to the hub. CySCs require Cnn, moesin (Moe), and dynein motor proteins for anaphase spindle repositioning. (c) Female GSCs also divide asymmetrically by orienting their spindle by anchoring one of the spindle poles to the spectrosome, which remains close to the hub-GSC interface throughout the cell cycle. Female GSCs require Hts protein (an integral component of the spectrosome/fusome) and cytoplasmic dynein to orient the spindle perpendicular to the hub.

hub cells, has been demonstrated to be required for stem cell maintenance. ${ }^{12}$

\section{Signaling Pathways in Male GSC and CySC Niche}

The hub supports self-renewal of GSCs and CySCs by secreting a short-range signaling ligand, unpaired (Upd), which activates the Janus kinase-signal transducer and activator of transcription (JAK-STAT) pathway in neighboring cells ${ }^{13,17,18}$ (Figure 2(c)).
GSCs and CySCs mutant for stat92E (STAT) or hop (JAK; encoded by the hopscotch gene) lose their ability to self-renew and instead differentiate. In addition, overexpression of Upd in early germ cells (GSCs or spermatogonia) or in early somatic cells (CySCs or cyst cells) causes overproliferation of undifferentiated, stem-like cells and results in tumor formation. Currently, the direct downstream targets of the JAKSTAT pathway that specify GSC identity are not well studied, although candidate genes have been described through microarray analysis. ${ }^{19}$ Recent studies have 
demonstrated that the transcriptional repressor zinc finger-homeodomain transcription factor $1(z f h-1)$ is a critical downstream target of the JAK-STAT pathway in CySCs. ${ }^{13}$ Strikingly, overexpression of Zfh-1 or forced expression of constitutively active JAK in $\mathrm{CySCs}$ resulted in overproliferation of not only CySCs but also GSCs. In contrast, ectopic expression of an active form of JAK tyrosine kinase in the germline did not cause massive proliferation of GSCs or CySCs. ${ }^{13}$ Furthermore, GSCs mutant for STAT can be maintained as long as CySCs have active Zfh1 , demonstrating CySCs as a critical component of the GSC niche. ${ }^{14}$ GSCs were suggested to require STAT activity only to correctly orient toward and to adhere to the hub cells. Zfh-1 appears to instruct GSC selfrenewal via TGF- $\beta$-dpp/gbb signaling. ${ }^{14}$ Decapentaplegic (dpp) and glass bottom boat (Gbb) are normally expressed in hub cells and CySCs, which ultimately leads to shutoff of Bam (Bag-of-marbles, a master regulator of differentiation) in germline, contributing to GSC self-renewal. ${ }^{20}$ However, interestingly, overexpression of dpp does not cause GSC tumors but leads to spermatogonial overproliferation, ${ }^{20-22}$ implying that there is an additional factor(s) downstream of Zfh-1 that function with transforming growth factorbeta (TGF- $\beta$ ) signaling to confer GSC identity.

\section{Orientation of the Spindle by the Positioning of Centrosomes in Male GSCs}

In the context of the niche signaling described above, the mitotic spindle is oriented perpendicular to the hub-GSC interface, leading to asymmetric division. Adherens junctions concentrated at the GSC cortex adjacent to the hub, along with ademomatous polyposis coli 2 (APC2), provide a polarity cue toward which GSCs orient throughout the cell cycle $^{4,23}$ (Figure 3(a)). In G1, the single centrosome in each GSC localizes near the cell cortex where the cell attaches to the hub. When the duplicated centrosomes are separated, one of the centrosomes stays next to the hub while the other migrates to the opposite side of the cell (Figure 3(a)). This stereotyped position of the centrosomes in turn orients the mitotic spindle perpendicular to the GSC-hub interface, leading to asymmetric division. Interestingly, the mother centrosome normally remains adjacent to the hub and is inherited by the GSC, whereas the newly duplicated centrosome migrates to the opposite side of the cell and is inherited by the $\mathrm{GB}^{24}$ (Figure 3(a)). This suggests that male GSCs retain the original (very old) centriole for a long time probably from the time the GSC population first arose during development. Indeed, centrosomes marked by a transient expression of a centriolar marker green fluorescent protein-pericentrin/AKAP450 (GFPPACT) during early development were retained in GSCs even in adult stage. ${ }^{24}$ The higher capacity of the mother centrosome to anchor astral microtubules may be the underlying cellular mechanism by which GSCs inherit the mother centrosome during division. In GSCs, centrosomes are separated unusually early, right after duplication, rather than at the G2/M transition: the mother centrosome appears to retain robust astral microtubules throughout the cell cycle, whereas the daughter centrosome migrating to the opposite side of the cell has few associated astral microtubules until late in G2, near the onset of mitosis. Consistently, positions of mother and daughter centrosomes as well as spindle orientation were randomized in GSCs mutant for centrosomin (cnn), which have severely impaired astral microtubules as a result of defective pericentriolar material. ${ }^{25-28}$ These results suggest that male GSCs have adopted cellular mechanisms that maintain stereotypical centrosome position and orient the mitotic spindle to tightly regulate the asymmetric outcome of stem cell divisions within the niche. Interestingly, a recent study demonstrated that mutants of DSas-4, a core component of centriole, normally orient mitotic spindle in male GSCs, despite the complete lack of centrioles (and thus centrosomes). ${ }^{29}$ Our unpublished study showed that, in DSas-4 mutant male GSCs, the spectrosome is located at the apical side of the GSC anchoring the spindle pole, which is reminiscent of spindle orientation mechanism in female GSCs ${ }^{30}$ suggesting that a parallel mechanism might compensate the loss of the centrosomes during asymmetric stem cell division.

\section{Anaphase Spindle Repositioning in CySCs}

The collaborative interaction of GSCs and CySCs sustains spermatogenesis. Because each GSC is encapsulated by a pair of CySCs, and a GB by a pair of cyst cells, CySCs must undergo two divisions for each GSC division on average. Recent studies have suggested that CySCs undergo consistent asymmetric divisions. ${ }^{15}$ However, GSCs and CySCs use strikingly different cellular mechanisms to accomplish asymmetric division: GSCs always orient their spindle perpendicular to the hub (Figure 3(a)), which reflects consistent centrosome polarization throughout multiple rounds of cell cycles, whereas CySCs reposition their spindle during anaphase (Figure 3(b)). CySCs enter mitosis with the spindle in a random orientation, but they reposition it so that one of the spindle poles is close to the hub cells during or near the onset of anaphase. ${ }^{15} \mathrm{CySC}$ spindles are very dynamic with both spindle poles rocking back and forth during metaphase, and then one spindle pole is quickly retracted to the hub-CySC interface around 
anaphase (Figure 3(b)). It remains unclear whether an asymmetry exists between two spindle poles as in male GSCs (i.e., poles containing mother vs daughter centrosomes, or poles with differential microtubule organizing activity). The detailed molecular mechanisms of spindle repositioning in CySCs are not yet completely clarified. Nevertheless, spindle repositioning in CySCs requires the following: (1) Cnn, an integral component of the centrosome, which is also required for GSC centrosome and spindle orientation; (2) dynein, the motor protein that pulls the spindle pole toward the cortical target site, similar to the case of Drosophila neuroblasts, where the dynein complex regulates spindle orientation; and (3) moesin (Moe), a cortex-actin cytoskeleton linking protein, which is essential for cell shape changes and spindle stability during mitosis in cultured cells. The molecular mechanisms that orient spindles are apparently different in male GSCs and CySCs: while GSCs require Cnn and Apc2 but not Moe, CySCs require Cnn and Moe but not Apc2 (Figure 3(a) and (b)). We can only speculate why GSCs and CySCs utilize such distinct cellular mechanisms to orient the spindle and divide asymmetrically. In contrast to GSCs that are round throughout the cell cycle, CySCs assume a flat and complex shape to wrap around GSCs, presumably reflecting the function of CySCs as a niche component for GSCs as described above. With such a flat and complex cell shape, maintaining spindle orientation from the beginning of mitosis might be difficult. Instead, CySCs might use spindle repositioning during mitosis, when cells become slightly rounder as is observed in cultured cells, to divide asymmetrically without compromising their role to wrap around GSCs during interphase. In spite of these differences, there is a similarity in the basic scheme of anchoring/pulling the spindle pole to the hub-GSC or hub-CySC interface in GSCs and CySCs: cortical network of adherens junction and actin cytoskeleton serving as a platform for pulling centrosomes through the function of motor proteins (Figure 3(a) and (b)). These studies illuminate the existence of distinct cellular mechanisms used by stem cells to divide asymmetrically, presumably depending on other factors, such as cell shape and tissue anatomy.

\section{Effect of Aging on Centrosome and Spindle Orientation in Male GSCs}

A decrease in stem cell number or activity may lead to tissue degeneration associated with age and disease. Indeed, age-dependent decrease in $U p d$ expression in the hub has been reported to contribute to GSC loss with advanced age. ${ }^{31}$ Similarly, female GSC number also declines with age because of changes to GSCs as well as to niche cells. ${ }^{32}$ Stem cell intrinsic and extrinsic changes appear to be general characteristics of stem cell aging, as is observed in mammalian stem cells. ${ }^{33-36}$ In addition, changes in stem cell orientation with respect to the niche, which precedes the decrease in GSC number, contribute to the decline in spermatogenesis: before the decrease in GSC number becomes significant, GSCs already slow down their proliferation due to increased centrosome misorientation. ${ }^{37}$ GSCs containing misoriented centrosomes accumulate progressively with age and these GSCs are arrested or delayed in the cell cycle and do not undergo mitosis. As a result, as Drosophila males age, a significant fraction of GSCs becomes arrested. Strikingly, this cell cycle arrest appears to be transient and GSCs re-enter the cell cycle upon correction of centrosome orientation. The latter implies that a novel checkpoint mechanism exists that blocks progression into mitosis unless a centrosome is properly situated next to the attachment to the hub.

Remarkably, many of the misoriented GSCs originate from the dedifferentiation of spermatogonia, a mechanism thought to be responsible for maintaining the stem cell population over extended periods of time. ${ }^{38,39}$ Throughout Drosophila adulthood, individual GSCs are lost at a certain rate. ${ }^{40,41}$ Dedifferentiation of partially differentiated spermatogonia to replace lost stem cells may be especially important in the male germline, because misoriented spindles, or symmetric stem cell division, are rarely observed in wild-type GSCs. Such dedifferentiated GSCs show a high incidence of misoriented centrosomes and undergo cell cycle arrest until proper centrosome orientation toward the hub is reestablished, increasing the average cell cycle length of GSCs, even if none of them are permanently arrested (i.e., quiescent). This might be correlated to the fact that germ cells that commit to differentiation do not inherit the 'very old' centrosome and that dedifferentiated GSCs have lost their 'very old' centrosome once they have committed to differentiation. ${ }^{42}$ Additional research is required to elucidate whether the returning germ cells anchor the relatively older centrosome near the junction between the GSCs.

Together, these studies reveal that stem cell aging, which ultimately leads to tissue and organismal aging, is a multifactorial process that involves both intrinsic and extrinsic changes.

\section{DROSOPHILA FEMALE GSCs}

\section{Architecture of the Female GSC Niche}

The Drosophila ovarian niche is composed of two kinds of somatic cell types: terminal filament $(\mathrm{TF})$ cells 
at the tip of the germarium (at the anterior tip of each ovariole, which is the egg-producing unit of the ovary) and cap cells at the base of the TF cells (Figure 2(b)). In each germarium, two to three GSCs are physically attached to five to seven cap cells via adherens junctions that contain both $\beta$-catenin and DE-cadherin (Figure 3(c)). Loss of either of these proteins from GSCs results in stem cell loss, suggesting that niche adhesion is essential for stem cell maintenance. ${ }^{43}$ Upon division of a GSC, one of the daughter cells stays associated with the niche and retains stem cell identity, while the other loses contact with the niche and becomes a cystoblast, which commits to differentiation. The cystoblast undergoes four additional rounds of transit amplifying divisions with incomplete cytokinesis to form a cyst of interconnected cells, which exits mitotic proliferation. Eventually, one of the 16 cells becomes the oocyte, whereas the other 15 undergo multiple rounds of endoreplication to produce mRNA and proteins to support oocyte production as nurse cells (Figure 2(b)). The ovarian GSC niche maintains yet another type of somatic cell type called escort stem cells (ESCs), which morphologically resemble male CySCs. ESCs are also tightly associated with cap cells and encapsulate GSCs with cytoplasmic extensions (Figure 2(b)). However, unlike CySCs in the testis, ESCs, as well as their daughter escort cells, appear to be normally quiescent, and developing germ cells move through ESCs and escort cells until they are encapsulated by follicle cells. ${ }^{44}$

\section{Signaling Pathways in Female GSC Niche}

Cap cells secrete two bone morphogenetic protein (BMP) ligands, Dpp and Gbb, which activate the type I and type II BMP receptors $\mathrm{Tkv}$ and Sax in GSCs, directly leading to transcriptional repression of Bam, a key differentiation promoting factor $^{45-47}$ (Figure 2(d)). In addition, an unknown signal regulated by $\mathrm{Piwi} / \mathrm{Yb}$ in $\mathrm{TFs}$ and cap cells is also involved in repressing Bam expression and thereby maintaining GSC self-renewal. ${ }^{48,49}$ It is unclear whether the BMP signal and the Piwiregulated signal from the cap cells intersect upstream to repress Bam expression or whether Piwi/Yb is involved in regulating BMP production in the niche. Nevertheless, forced expression of Bam in GSCs causes them to differentiate and loss of Bam results in cystoblasts that continue to proliferate like stem cells, leading to the formation of an ovarian tumor. ${ }^{41}$ Similar to male GSCs, JAK-STAT signaling plays a critical role in ovarian niche function, and acts within both cap cells and ESCs. Recent studies have shown that JAK-STAT signaling in cap cells positively regulates dpp expression, thereby contributing to GSC self-renewal. ${ }^{50,51}$ ESCs also require JAK-STAT signals to specify ESC and GSC identity. Removal of STAT from ESCs results in rapid loss of GSCs, whereas overexpression of Upd (JAK-STAT ligand) in ESCs results in an increase in numbers of both ESCs and GSCs, leading to occasional formation of germline tumors. Similar to the idea that male GSCs require signals from both the hub and CySCs for self-renewal, female GSCs seem to not only require the Dpp signal from cap cells, but also an additional unknown signal provided by the ESCs, which is mediated by the activation of JAK-STAT signaling within ESCs ${ }^{52}$ (Figure 2(d)).

\section{Orientation of the Spindle by the Spectrosome in Female GSCs}

Like male GSCs, female GSCs normally undergo asymmetric cell division to maintain the balance between the number of stem cells and differentiating cells. However, female GSCs use a different cellular mechanism than male GSCs to orient their mitotic spindle perpendicular to the niche surface. Although the mitotic spindle in male GSCs is set up by the stereotypical positioning of the centrosomes as described earlier (Figure 3(a)), the spindle in the female GSCs is oriented by anchoring one of the spindle poles to the spectrosome, a prominent spectrin-rich spherical organelle in the cytoplasm of GSCs in the ovary ${ }^{30,53}$ (Figure 3(c)). Consistent with this, eliminating the spectrosome in female GSCs leads to randomized spindle orientation. ${ }^{30}$ Cytoplasmic dynein has been reported to be involved in the attachment of the spindle pole to the spectrosome. ${ }^{54}$ In agreement with the fact that the spectrosome anchors the mitotic spindle in female GSCs, asymmetric localization of the spectrosome near the hub-GSC interface persists throughout the cell cycle of GSCs, whereas centrosome positions are reported to be random during interphase of GSCs and are not required for spindle orientation. ${ }^{55}$ In contrast, in male GSCs, spectrosome localization shows no consistent localization with respect to the hub cells in interphase, ${ }^{4}$ and centrosome/spindle orientation is not perturbed in the absence of the spectrosome (our unpublished results).

Female GSCs are sporadically lost from the niche with a half-life of approximately $4-5$ weeks. ${ }^{41}$ In contrast to male GSCs, which rarely have spindles parallel to the hub cells, female GSCs can divide with their mitotic spindles parallel to cap cells, resulting in symmetric stem cell division, with both daughters maintaining niche contact and stem cell identity. ${ }^{56}$ Female germ cells also have the potential to undergo dedifferentiation. ${ }^{39}$ Thus, both mechanisms, dedifferentiation and symmetric division, may contribute to 
maintaining GSC number in the ovary. These results illuminate common and distinct molecular mechanisms underlying female versus male GSC asymmetric divisions.

\section{OTHER STEM CELL SYSTEMS: VARIATION ON A THEME}

In many cases, the molecular details of spindle orientation and its reasoning (e.g., which cells are the niche components, which signaling pathway is employed by those cells, and the implication of spindle orientation with respect to those niche cells) are not fully understood. However, consistent spindle orientation is observed in various stem cell types, implying that spindle orientation serves as a regulatory mechanism for stem cell behavior. However, there are also stem cell types that do not show consistent spindle orientation. In this article, we summarize such examples of stem cells, where spindle orientation and/or niche are characterized relatively well, even if the entire picture is not clear.

\section{Drosophila ISCs}

The adult Drosophila midgut contains multipotent intestinal stem cells (ISCs), which maintain the intestinal epithelium by generating enterocytes as well as enteroendocrine cells. ${ }^{57-59}$ Notch signaling, with the Delta ligand coming from ISCs, regulates the differentiation of ISC daughters. ISCs are found along the basement membrane within clusters of two or three diploid cells that are dispersed among polyploid enterocytes lining the intestine. ISCs appear to divide asymmetrically with a non-random spindle orientation, resulting in ISCs typically maintaining a much greater surface area of contact with the basement membrane compared with their differentiating daughters. ${ }^{58}$ It was reported that wingless ligand from muscle cells, which are found immediately underneath the basement membrane, activate wingless signaling within ISCs for their self-renewal. ${ }^{60}$ It might be that the observed spindle orientation within ISCs leads to asymmetric inheritance of the contact with the wingless source. However, the mechanism that regulates orientation of the mitotic spindle has not yet been characterized.

\section{Mammalian ISCs}

Mammalian ISCs have been proposed to be located within or at the fourth position ( +4 cells) from the bottom of the crypt. ${ }^{61}$ Recent studies have shown that crypt base columnar (CBC) cells specifically expressing Leucine-rich repeat-containing G-protein coupled receptor 5 (Lgr5) located at crypt bottoms represent ISCs. Indeed, a single Lgr5+ stem cell can form a long-lived, self-renewing 'minigut' or 'organoid', when cultured under appropriate conditions. ${ }^{62}$ It was demonstrated that terminally differentiated Paneth cells, which are derived from Lgr5+ stem cells, constitute the niche for the stem cells in the intestinal crypt. ${ }^{63}$ Paneth cells secrete epidermal growth factor (EGF), transforming growth factor-alpha (TGF- $\alpha$ ), Wnt3, and the Notch ligand Dll4, which are all required for stem cell maintenance in intestinal crypt. Consistently, co-culture of Lgr5+ stem cells with already differentiated Paneth cells produced organoids with a better efficiency. ${ }^{63}$ Another study showed that Bmi$1+$ cells at the fourth position of the crypt represent ISCs. ${ }^{64}$ The relationship between $\operatorname{Lgr} 5+$ and Bmi-1+ cells is currently unknown.

Spindle orientation of ISCs is currently controversial. While a report suggests that cells within the Lgr5+ stem cell compartment tend to align their mitotic spindle perpendicular to the lumen of the crypt, which is randomized in apc mutant crypts, ${ }^{65}$ a more recent report documented random spindle orientation in ISCs. ${ }^{66}$ Clonal analysis of Lgr5+ ISCs demonstrated that this population is maintained by neutral competition between symmetrically dividing stem cells. ${ }^{67}$ Furthermore, the major niche component Paneth cells intermingle with ISCs/CBC, ${ }^{63}$ making it difficult to imagine how the spindle orientation perpendicular to the lumen contributes to asymmetric stem cell division. These data are inconsistent with the idea that oriented spindle contributes to asymmetric ISC divisions. Therefore, reported spindle orientation in Lgr $5+$ cells might reflect other biological process(es) than asymmetric stem cell division.

\section{Mammalian Skin Stem Cells}

The stem cells in developing epidermis reside within the innermost (basal) layer of the epithelium, which rests upon the basement membrane. Skin stem cells rely upon signals from the basement membrane to retain stem cell identity. They differentiate as they exit this niche and move to suprabasal locations. During early stages of embryonic development, most spindles are oriented parallel to the basement membrane in the single-layered epidermis (Figure 4(a)). Later, starting at embryonic day (E)14 coincident with stratification, most spindles are oriented perpendicularly, generating one basal and one suprabasal cell ${ }^{68}$ (Figure $\left.4(\mathrm{~b})\right)$. This perpendicular, and thus asymmetric, division is indeed required for stratification of the epidermis. ${ }^{69}$

Spindle orientation is set up by evolutionarily conserved apical-basal polarity determinants such 


\section{(a) Early embryonic development}

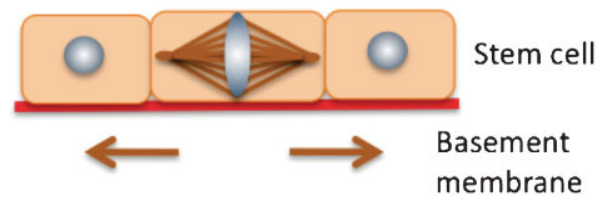

\section{(b) Stratification during morphogenesis}

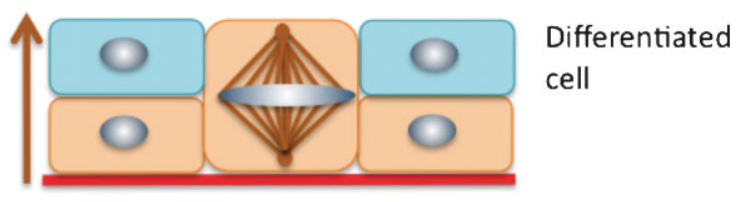

FIG URE 4 | Asymmetric cell division in mammalian epithelial stem cells. (a) During embryonic development, most cell divisions are symmetric in order to maintain the epithelium as a single layer. (b) During stratification, majority of the cell divisions become asymmetric, such that the mitotic spindle is perpendicular to the basement membrane. Different cell fate determinants are segregated unequally between the stem cell (basal) and differentiated (suprabasal) cell.

as partitioning defective-3 (Par-3), atypical protein kinase $\mathrm{C}$ (aPKC), Leucine-Glycine-Asparagine tripeptides in its N-terminal region (LGN, also known as GPSM2), and inscuteable (INSC), which interact with components of the mitotic spindle such as nuclear mitotic apparatus 1 (NuMA1) and the dynactin component p150Glued, to govern spindle orientation. Interestingly, the polarized localization of these proteins relies on integrins and cadherins, linking intracellular polarity to tissue polarity. Furthermore, as in the Drosophila neuroblast, asymmetric division of these stem cells involves the Notch signaling pathway.., 69

Recent work has demonstrated that each epidermal cell can undergo both asymmetric and symmetric divisions, which are regulated by expression of INSC and the localization of NuMA. ${ }^{70}$ This has provided direct evidence that each stem cell can switch between asymmetric and symmetric divisions, at least in this system, instead of becoming distinct subpopulations that undergo only symmetric or asymmetric divisions.

While the evidence for stem cell population and their asymmetric cell division via spindle orientation appears to be solid, stem cell behavior in adult epidermis is less known. Clonal analysis showed that a single population, referred to as committed progenitors, which divide and self-renew/differentiate stochastically, maintains homeostasis of adult epidermis. ${ }^{71}$ This rather resembles to the case of intestinal crypt stem cells, which maintains the population by neutral competition of symmetrically dividing stem cells. Thus it is to be determined whether spindle orientation plays a role in the homeostasis of adult epidermis.

\section{Mouse Satellite Cells}

Satellite cells, the stem cells for muscle fibers, play an important role in maintaining homeostasis of muscle tissue and promoting muscle repair after injury. Satellite cells reside beneath a basal lamina, adjacent to mature myofibers. These stem cells are maintained by the signals from the host muscle fiber. ${ }^{72,73}$ In addition, anchoring to the basal lamina, which mainly consists of laminin, collagen, and proteoglycans, is vital for maintenance of stem cell identity. ${ }^{74}$ Satellite cells are normally quiescent but can be induced to enter the cell cycle upon injury. Recent studies have demonstrated that a Pax7+Myf5- satellite stem cell can asymmetrically generate another Pax $7+$ Myf5 - stem cell and a Pax7+Myf5+ committed daughter cell. ${ }^{75}$ The basal lamina side of a satellite cell expresses integrin $\alpha 7 \beta 1$ receptors that interact with laminin, whereas the apical side expresses the cell adhesion molecule M-cadherin that anchors the satellite cell to and transduces signals from the myofibers. ${ }^{76,77}$ As a result, a planar spindle orientation generates two daughter cells that are exposed to both apical and basal signals, resulting in two daughter cells with stem cell fate. In contrast, an apical-basal spindle orientation produces two daughter cells so that one daughter remains in contact with the basal lamina and adopts a stem cell fate and the other daughter that loses basal lamina contact adopts a committed fate. ${ }^{75}$ In the case of acute injuries that require rapid expansion of satellite cells from neighboring uninjured muscle, satellite cells have been shown to predominately divide symmetrically. ${ }^{78,79}$ The molecular mechanisms that establish satellite cell polarity and the mitotic spindle orientation remain to be determined.

\section{Caenorhabditis elegans GSCs: No Spindle Orientation}

C. elegans GSCs are an interesting and illuminating example where the contribution and location of the stem cell niche are clear, yet the spindle is not oriented with respect to the niche. In C. elegans, the distal tip cell (DTC) at the distal end of the adult gonad creates the niche for GSCs. The DTC signals to the germline via the Notch signaling pathway mediated by Delta/Serrate-like ligands LAG-2 and APX-1, 
which are produced by the DTC and the germline proliferation-1/Notch receptor present on the surface of the germ cells. ${ }^{80,81}$ Accordingly, daughters in close proximity to the DTC retain stem cell identity and daughters further from the DTC initiate differentiation. Unlike Drosophila GSC divisions, which are oriented perpendicular to the niche as described above, C. elegans germ cells do not reveal any bias in orientation along the distal-proximal axis of the gonad. ${ }^{82}$ Thus it appears that the number of C. elegans GSCs is controlled solely by juxtaposition to the niche component cells, instead of by the asymmetric outcome of each GSC division. Importantly, this indicates that we might not necessarily be able to 'guess' stem cell populations within the tissue based on their biased spindle orientation.

\section{The Spindle Orientation Mechanism is Conserved in Systems that Do Not Rely on the Niche}

Drosophila embryonic and larval neuroblasts undergo asymmetric divisions to generate a self-renewed neuroblast and a GMC, which divides once more to produce two cells that terminally differentiate. In Drosophila neuroblasts, the proteins Par-3, Par6, aPKC, INSC, Pins, G $\alpha$ i, and Mud accumulate on the apical side of the cell cortex and are preferentially inherited by the neuroblast. ${ }^{5,83}$ These proteins are not thought to influence cell fate directly. Instead, they induce the asymmetric localization of cell fate determinants such as Numb, Pon, Miranda, Prospero, and Brat to the basal side and their segregation to the GMC. Remarkably, recent liveimaging studies have revealed that the outcome of neuroblast division (i.e., symmetric vs asymmetric) is governed by the ratio of apical to basal determinants, but apical determinants dominate basal determinants under normal expression levels. ${ }^{84}$ The asymmetric distribution of apical Par proteins sets up the axis of polarity in neuroblasts. ${ }^{85}$ As expected, in par mutants, mitotic spindles are oriented randomly and cell fate determinants are uniformly distributed around the cell cortex.

Interestingly, recent studies have suggested that centrosome asymmetry is utilized for asymmetric division of Drosophila neuroblasts. ${ }^{86,87}$ One centrosome remains bound to the apical cortex, whereas the other moves to the other side of the cell to set up the desired spindle orientation. In contrast to Drosophila male GSCs, however, the daughter centriole is inherited by the neuroblast upon division by programed inactivation of the mother centrosome and activation of the daughter centrosome during each cell cycle. ${ }^{88,89}$ Perturbation in centrosome function (in either cnn or DSas-4 mutant) led to symmetric neuroblast divisions. ${ }^{90,91}$ Asymmetric neuroblast division was also perturbed when centrosome numbers were amplified. ${ }^{92}$ However, these conditions led to only a mild increase in neuroblast number (as is the case for $\mathrm{cnn}$ mutant $\mathrm{GSCs}^{4}$ ). Together, while these data demonstrate an important contribution of cell biological differences between mother and daughter centrosomes, it also argues against a fate-determining role of mother or daughter centrosome.

Radial glial progenitor cells in the cerebral cortex in mouse embryos predominantly undergo asymmetric divisions after the onset of neurogenesis. During their asymmetric division, spindle orientation is tightly regulated so that only one daughter cell inherits the apical cortex containing Par-3, similar to Drosophila neuroblasts. ${ }^{93}$ Interestingly, as in Drosophila male GSCs, radial glial progenitor cells preferentially inherit the mother centrosome upon division, ${ }^{94}$ which suggests evolutionary conservation of centrosome asymmetry as a strategy for asymmetric division. The loss of ninein, a mature centriole-specific protein, results in loss of radial glial progenitor cells, leading the authors to suggest that the inheritance of the mother centrosome is critical for the maintenance of stem cells (radial glial progenitor cells). ${ }^{94}$ However, it remains possible that the loss of centrosome function, rather than mother centrosome per se, leads to defective maintenance of radial glial progenitor cells. In case of male GSCs, spermatogonia, which had lost their original mother centrosome upon initiation of differentiation, can revert back to GSC by dedifferentiation, suggesting that GSC identity do not absolutely rely on the existence of mother centrosome. ${ }^{37}$ Nonetheless, studies on congenital microcephaly in humans have identified mutations in genes encoding centrosomal proteins, ${ }^{95,96}$ demonstrating the importance of centrosomal function in the maintenance of neural progenitor cells.

\section{FURTHER IMPLICATION OF SPINDLE ORIENTATION: BEYOND FATE DETERMINATION}

As reviewed above, coordination of fate determinants and spindle orientation is a common mechanism for asymmetric stem cell division. Other lines of studies have implied that the spindle might be utilized to asymmetrically segregate other factors, which are not fate determinants per se, but related to or associated with cell fate as a stem cell or a differentiating daughter. Such factors might behave like a 
(a)

Second division

Immortal strand Random strand

segregation

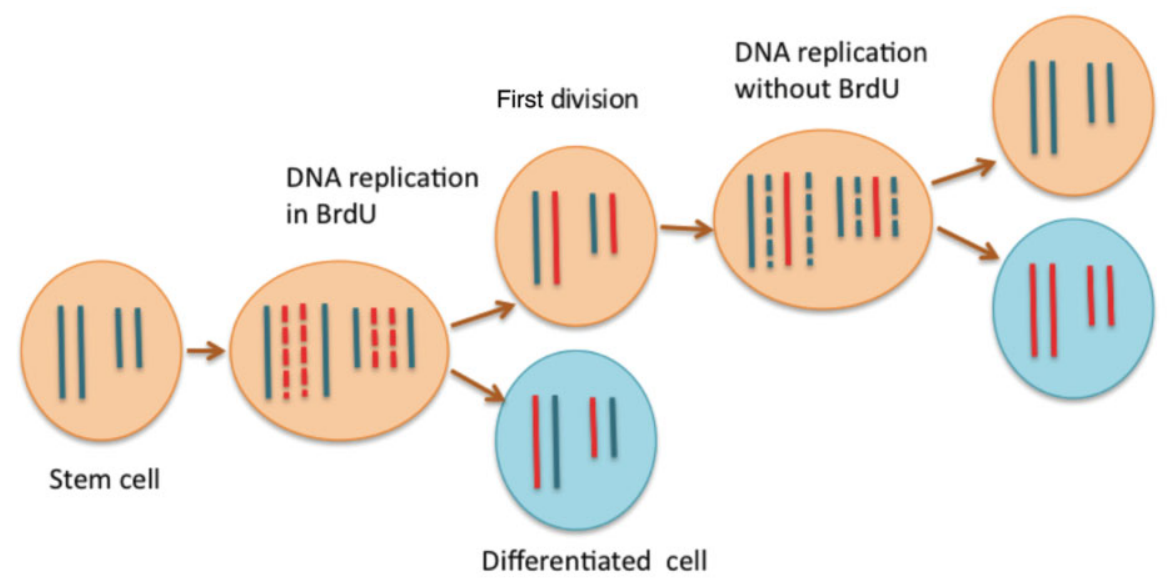

segregation

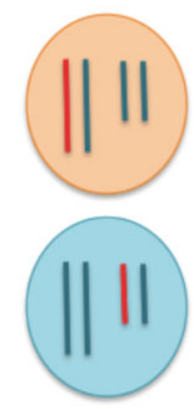

\begin{tabular}{l|l} 
|- unlabeled chromosome & - BrdU-labeled chromosome
\end{tabular}

(b)

(1) Intestinal cells

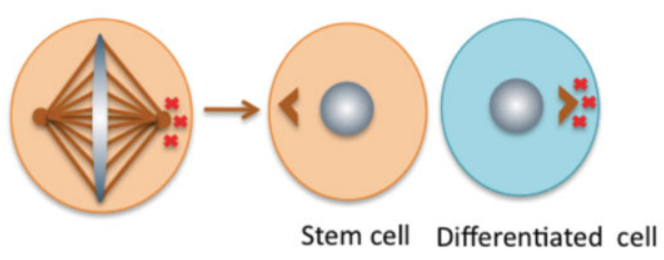

* - aggresomes
(2) Fly neuroblast
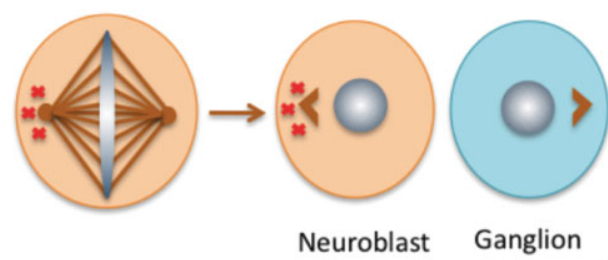

mother cell

FIGURE 5 | Mitotic asymmetries of DNA and protein aggregates. (a) According to the immortal strand model, stem cells asymmetrically segregate newly synthesized bromodeoxyuridine (BrdU)-positive strands into differentiating daughter cells, such that stem cell retains only the unlabeled older strands. On the other hand, if chromosomes are randomly segregated, then both the stem cell and the differentiating daughter cell randomly inherit the BrdU-labeled chromosomes until the entire BrdU label is diluted stochastically over time. (b) Aggregates of misfolded proteins accumulate near one of the centrosomes and are asymmetrically inherited during mitosis such that majority of the aggresomes are retained in the short-lived progeny.

hitchhiker that utilizes spindles to be segregated asymmetrically, for ultimate purposes such as protecting stem cells.

\section{Asymmetric Inheritance of Chromosome Strands during Asymmetric Cell Division}

The 'immortal strand hypothesis' (ISH) has been proposed as a mechanism by which adult stem cells might limit accumulation of mutations arising from errors during DNA replication. The ISH proposes that adult stem cells might retain older (immortal) DNA strands during asymmetric cell divisions, thereby excluding all replication-induced mutations to the differentiating daughter cells. ${ }^{97}$ This hypothesis has been intensively studied in recent years in various stem cell populations. In these studies, a short pulse of bromodeoxyuridine (BrdU) during $S$ phase was often used to label newly synthesized DNA strands followed by a chase period without BrdU, during which the segregation of BrdUlabeled chromosomes was monitored (Figure 5(a)). These BrdU pulse-chase experiments have supported the ISH in some stem cell systems (mammary epithelial cells, ${ }^{98}$ skeletal muscle satellite cells, ${ }^{78,79}$ adult neural stem cells, ${ }^{99,100}$ and Drosophila female GSCs ${ }^{101}$ ), but not in other systems (epidermal basal cells, ${ }^{102}$ hair follicle stem cells, ${ }^{103}$ embryonic neocortical precursor cells, ${ }^{100}$ mouse hematopoietic stem cells, ${ }^{104}$ 
and Drosophila male GSCs ${ }^{105}$ ). In some cases, there have been seemingly contradictory reports on the same/similar cell types (mouse intestinal crypt stem cells $\left.{ }^{65,66}\right)$. In most systems, whether stem cells always divide asymmetrically, or switch between asymmetric and symmetric divisions is unclear. In addition, the simultaneous visualization of daughter cell fates (stem cells vs differentiating daughter) and BrdU segregation patterns is challenging in many systems. These complications have hampered a clear-cut interpretation of the experimental data, sometimes leading to contradictory interpretations even with the same stem cell type. Therefore, explicitly addressing the ISH awaits further investigation. A recent work has adapted Chromosome orientation-fluorescence in situ hybridization technique, in which strand-specific probes hybridize only to the older strands of chromosomes that had not incorporated BrdU to study chromosome strand segregation. ${ }^{106}$ They identified 'plus' and 'minus' DNA template strands of mouse chromosomes by using probes specific for centromeric and telomeric repeats. With this method, they observed significant non-random chromosome segregation in a subset of colon crypt epithelial cells, whereas chromosome strands appeared to be randomly distributed in cultured lung fibroblasts and embryonic stem cells.

Recently, it was speculated that the mother centrosome, which is stereotypically retained within some stem cells such as Drosophila male GSCs and mouse radial glial progenitors, might be used as a means to anchor the immortal DNA strand. ${ }^{107}$ However, our recent work has demonstrated that Drosophila male GSCs do not follow the immortal strand model-although their centrosome segregation is asymmetric. Therefore, the 'immortal' mother centrosome does not necessarily lead to immortal DNA strand segregation. ${ }^{105}$

\section{Asymmetric Inheritance of Misfolded Proteins}

Aging at the cellular level is generally characterized by a decline in cell function and has been correlated with accumulation of damaged or misfolded proteins. An excess of misfolded proteins forms aggresomes, which cannot be degraded by the proteasome. Live-imaging studies of human mitotic cells have demonstrated that experimentally induced aggresomes accumulate near one of the centrosomes and are thus inherited by only one daughter. ${ }^{108}$ Rujano et al. showed that such aggresomes are inherited by only one daughter upon division by associating with the centrosomes. ${ }^{109}$ Crypt stem cells of the small intestine from patients with a protein folding disease are devoid of aggregates, even though differentiated cells accumulated the misfolded proteins, leading the authors to suggest that asymmetric segregation of aggresomes to the differentiating daughters is the mechanism to protect stem cells (Figure 5(b)). However, it was shown that Lgr5+ ISCs divide symmetrically as described earlier, ${ }^{67}$ and thus it is not clear how aggresomes might be asymmetrically segregated during mitosis. Yet, asymmetric segregation of aggresomes and symmetric ISC divisions are not necessarily mutually exclusive: now that definitive stem cell markers such as Lgr5 and Bmi-1 are available, aggresome segregation in ISC and differentiating cell compartments can be revisited with precision. In the same report, it was also shown that Drosophila neuroblasts engineered to express a fragment of huntingtin display a polarized distribution of huntingtin in mitosis, although in this case aggresomes segregate into the neuroblast, leaving the GMC free of aggregates. ${ }^{109}$ This led to the speculation that cells with a longer life-time (which are neurons rather than neuroblasts in the case of the Drosophila neuronal lineage) are protected from accumulation of misfolded proteins (Figure 5(b)).

\section{CONCLUSION}

In this article, we reviewed the mechanism of asymmetric stem cell division by means of spindle orientation. Accumulating knowledge illuminates the importance of spindle orientation as a common strategy conserved in many stem cells. Spindle orientation in the context of tissue anatomy is precisely regulated such that stem cell division occurs in a desired way (asymmetric vs symmetric division) for tissue development and homeostasis. The gap between our current understanding of stem cell-specific factors such as master transcription factors of stemness and our current understanding of generic cellular components such as microtubules and cell-cell junctions has only begun to be filled. We foresee exciting years to come during which this gap will be filled, bringing about a comprehensive understanding of how the stem cell program controls its cellular components to achieve their remarkable function. 


\section{REFERENCES}

1. Morrison SJ, Kimble J. Asymmetric and symmetric stem-cell divisions in development and cancer. Nature 2006, 441:1068-1074.

2. Rando TA. Stem cells, ageing and the quest for immortality. Nature 2006, 441:1080-1086.

3. Yamashita YM, Yuan H, Cheng J, Hunt AJ. Polarity in stem cell division: asymmetric stem cell division in tissue homeostasis. Cold Spring Harb Perspect Biol 2010, 2: a001313.

4. Yamashita YM, Jones DL, Fuller MT. Orientation of asymmetric stem cell division by the APC tumor suppressor and centrosome. Science 2003, 301: 1547-1550.

5. Knoblich JA. Mechanisms of asymmetric stem cell division. Cell 2008, 132:583-597.

6. Hirata J, Nakagoshi H, Nabeshima Y, Matsuzaki F. Asymmetric segregation of the homeodomain protein Prospero during Drosophila development. Nature 1995, 377:627-630.

7. Knoblich JA, Jan LY, Jan YN. Asymmetric segregation of Numb and Prospero during cell division. Nature 1995, 377:624-627.

8. Spana EP, Doe CQ. The prospero transcription factor is asymmetrically localized to the cell cortex during neuroblast mitosis in Drosophila. Development 1995, 121:3187-3195.

9. Kaltschmidt JA, Davidson CM, Brown NH, Brand $\mathrm{AH}$. Rotation and asymmetry of the mitotic spindle direct asymmetric cell division in the developing central nervous system. Nat Cell Biol 2000, 2:7-12.

10. Rebollo E, Roldan M, Gonzalez C. Spindle alignment is achieved without rotation after the first cell cycle in Drosophila embryonic neuroblasts. Development 2009, 136:3393-3397.

11. Hardy RW, Tokuyasu KT, Lindsley DL, Garavito M. Germinal proliferation center in the testis of Drosophila melanogaster. J Ultrastruct Res 1979, 69:180-190.

12. Voog J, D'Alterio C, Jones DL. Multipotent somatic stem cells contribute to the stem cell niche in the Drosophila testis. Nature 2008, 454:1132-1136.

13. Leatherman JL, DiNardo S. Zfh-1 controls somatic stem cell self-renewal in the Drosophila testis and nonautonomously influences germline stem cell selfrenewal. Cell Stem Cell 2008, 3: 44-54.

14. Leatherman JL, Dinardo S. Germline self-renewal requires cyst stem cells and stat regulates niche adhesion in Drosophila testes. Nat Cell Biol 2010, 12: 806-811.

15. Cheng J, Tiyaboonchai A, Yamashita YM, Hunt AJ. Asymmetric division of cyst stem cells in Drosophila testis is ensured by anaphase spindle repositioning. Development 2011, 138:831-837.
16. Fuller MT. Spermatogenesis. In: Bate M, Arias AM, eds. The Development of Drosophila melanogaster. Cold Spring Harbor, NY: Cold Spring Harbor Laboratory Press; 1993, 71-147.

17. Kiger AA, Jones DL, Schulz C, Rogers MB, Fuller MT. Stem cell self-renewal specified by JAK-STAT activation in response to a support cell cue. Science 2001, 294:2542-2545.

18. Tulina N, Matunis E. Control of stem cell self-renewal in Drosophila spermatogenesis by JAK-STAT signaling. Science 2001, 294:2546-2549.

19. Terry NA, Tulina N, Matunis E, DiNardo S. Novel regulators revealed by profiling Drosophila testis stem cells within their niche. Dev Biol 2006, 294:246-257.

20. Kawase E, Wong MD, Ding BC, Xie T. Gbb/Bmp signaling is essential for maintaining germline stem cells and for repressing bam transcription in the Drosophila testis. Development 2004, 131:1365-1375.

21. Schulz C, Kiger AA, Tazuke SI, Yamashita YM, Pantalena LC, Jones DL, Wood CG, Fuller MT. A misexpression screen reveals effects of bag-of-marbles and TGF beta class signaling on the Drosophila male germline stem cell lineage. Genetics 2004, 167:707-723.

22. Shivdasani AA, Ingham PW. Regulation of stem cell maintenance and transit amplifying cell proliferation by TGF-beta signaling in Drosophila spermatogenesis. Curr Biol 2003, 13:2065-2072.

23. Inaba M, Yuan HB, Salzmann V, Fuller MT, Yamashita YM. E-cadherin is required for centrosome and spindle orientation in Drosophila male germline stem cells. Plos One 2010, 5:e12473. doi:10.1371/journal.pone.0012473.

24. Yamashita YM, Mahowald AP, Perlin JR, Fuller MT. Asymmetric inheritance of mother versus daughter centrosome in stem cell division. Science 2007, 315: $518-521$.

25. Megraw TL, Li KJ, Kao LR, Kaufman TC. The centrosomin protein is required for centrosome assembly and function during cleavage in Drosophila. Development 1999, 126:2829-2839.

26. Megraw TL, Kao LR, Kaufman TC. Zygotic development without functional mitotic centrosomes. Curr Biol 2001, 11:116-120.

27. Megraw TL, Kilaru S, Turner FR, Kaufman TC. The centrosome is a dynamic structure that ejects PCM flares. J Cell Sci 2002, 115:4707-4718.

28. Vaizel-Ohayon D, Schejter ED. Mutations in centrosomin reveal requirements for centrosomal function during early Drosophila embryogenesis. Curr Biol 1999, 9:889-898.

29. Riparbelli MG, Callaini G. Male gametogenesis without centrioles. Dev Biol 2011, 349:427-439.

30. Deng W, Lin HF. Spectrosomes and fusomes anchor mitotic spindles during asymmetric germ cell divisions 
and facilitate the formation of a polarized microtubule array for oocyte specification in Drosophila. Dev Biol 1997, 189:79-94.

31. Boyle M, Wong C, Rocha M, Jones DL. Decline in self-renewal factors contributes to aging of the stem cell niche in the Drosophila testis. Cell Stem Cell 2007, 1:470-478.

32. Pan L, Chen SY, Weng CJ, Call G, Zhu DX, Tang H, Zhang N, Xie T. Stem cell aging is controlled both intrinsically and extrinsically in the Drosophila ovary. Cell Stem Cell 2007, 1:458-469.

33. Conboy IM, Conboy MJ, Smythe GM, Rando TA. Notch-mediated restoration of regenerative potential to aged muscle. Science 2003, 302:1575-1577.

34. Rossi DJ, Bryder D, Zahn JM, Ahlenius H, Sonu $\mathrm{R}$, Wagers AJ, Weissman IL. Cell intrinsic alterations underlie hematopoietic stem cell aging. Proc Natl Acad Sci U S A 2005, 102:9194-9199.

35. Molofsky AV, Slutsky SG, Joseph NM, He S, Pardal R, Krishnamurthy J, Sharpless NE, Morrison SJ. Increasing p16INK4a expression decreases forebrain progenitors and neurogenesis during ageing. Nature 2006, 443:448-452.

36. Conboy IM, Rando TA. Aging, stem cells and tissue regeneration: lessons from muscle. Cell Cycle 2005, 4:407-410.

37. Cheng J, Turkel N, Hemati N, Fuller MT, Hunt AJ, Yamashita YM. Centrosome misorientation reduces stem cell division during ageing. Nature 2008, 456:599-604.

38. Brawley C, Matunis E. Regeneration of male germline stem cells by spermatogonial dedifferentiation in vivo. Science 2004, 304:1331-1334.

39. Kai T, Spradling A. Differentiating germ cells can revert into functional stem cells in Drosophila melanogaster ovaries. Nature 2004, 428:564-569.

40. Wallenfang MR, Nayak R, DiNardo S. Dynamics of the male germline stem cell population during aging of Drosophila melanogaster. Aging Cell 2006, 5:297-304.

41. Xie T, Spradling AC. decapentaplegic is essential for the maintenance and division of germline stem cells in the Drosophila ovary. Cell 1998, 94:251-260.

42. Yamashita YM, Fuller MT. Asymmetric centrosome behavior and the mechanisms of stem cell division. J Cell Biol 2008, 180:261-266.

43. Song XQ, Zhu CH, Doan C, Xie T. Germline, stem cells anchored by adherens junctions in the Drosophila ovary niches. Science 2002, 296:1855-1857.

44. Morris L, Spradling AC. Long-term live imaging provides new insight into stem cell regulation and germline-soma regulation in the Drosophila ovary. Development 2011, 1:2207-2215.

45. Chen DH, McKearin D. Dpp signaling silences bam transcription directly to establish asymmetric divisions of germline stem cells. Curr Biol 2003, 13:1786-1791.
46. Chen DH, McKearin DM. A discrete transcriptional silencer in the bam gene determines asymmetric division of the Drosophila germline stem cell. Development 2003, 130:1159-1170.

47. Song XQ, Wong MD, Kawase E, Xi RW, Ding BC, McCarthy JJ, Xie T. Bmp signals from niche cells directly repress transcription of a differentiationpromoting gene, bag of marbles, in germline stem cells in the Drosophila ovary. Development 2004, 131: 1353-1364.

48. Chen D, McKearin D. Gene circuitry controlling a stem cell niche. Curr Biol 2005, 15:179-184.

49. Szakmary A, Cox DN, Wang Z, Lin H. Regulatory relationship among piwi, pumilio, and bag-of-marbles in Drosophila germline stem cell self-renewal and differentiation. Curr Biol 2005, 15:171-178.

50. Wang L, Li Z, Cai Y. The JAK/STAT pathway positively regulates DPP signaling in the Drosophila germline stem cell niche. J Cell Biol 2008, 180: $721-728$.

51. Lopez-Onieva L, Fernandez-Minan A, Gonzalez-Reyes A. Jak/Stat signalling in niche support cells regulates dpp transcription to control germline stem cell maintenance in the Drosophila ovary. Development 2008, $135: 533-540$.

52. Decotto E, Spradling AC. The Drosophila ovarian and testis stem cell niches: similar somatic stem cells and signals. Dev Cell 2005, 9:501-510.

53. Roth S, Lynch JA. Symmetry breaking during Drosophila oogenesis. Cold Spring Harb Perspect Biol 2009, 1:a001891.

54. McGrail M, Hays TS. The microtubule motor cytoplasmic dynein is required for spindle orientation during germline cell divisions and oocyte differentiation in Drosophila. Development 1997, 124:2409-2419.

55. Stevens NR, Raposo AASF, Basto R, St Johnston D, Raff JW. From stem cell to embryo without centrioles. Curr Biol 2007, 17:1498-1503.

56. Xie T, Spradling AC. A niche maintaining germ line stem cells in the Drosophila ovary. Science 2000, 290:328-330.

57. Ohlstein B, Spradling A. The adult Drosophila posterior midgut is maintained by pluripotent stem cells. Nature 2006, 439:470-474.

58. Ohlstein B, Spradling A. Multipotent Drosophila intestinal stem cells specify daughter cell fates by differential Notch signaling. Science 2007, 315:988-992.

59. Micchelli CA, Perrimon N. Evidence that stem cells reside in the adult Drosophila midgut epithelium. Nature 2006, 439:475-479.

60. Lin G, Xu N, Xi R. Paracrine wingless signalling controls self-renewal of Drosophila intestinal stem cells. Nature 2008, 455:1119-1123.

61. Cheng H, Leblond CP. Origin, differentiation and renewal of 4 main epithelial-cell types in mouse small 
intestine 5. Unitarian theory of origin of 4 epithelialcell types. Am J Anat 1974, 141:537-561.

62. Sato T, Vries RG, Snippert HJ, van de Wetering M, Barker N, Stange DE, van Es JH, Abo A, Kujala P, Peters PJ, et al. Single Lgr5 stem cells build cryptvillus structures in vitro without a mesenchymal niche. Nature 2009, 459:262-265.

63. Sato T, van Es JH, Snippert HJ, Stange DE, Vries RG, van den Born M, Barker N, Shroyer NF, van de Wetering M, Clevers $\mathrm{H}$. Paneth cells constitute the niche for Lgr5 stem cells in intestinal crypts. Nature 2011, 469:415-418.

64. Sangiorgi E, Capecchi MR. Bmi1 is expressed in vivo in intestinal stem cells. Nat Genet 2008, 40:915-920.

65. Quyn AJ, Appleton PL, Carey FA, Steele RJC, Barker N, Clevers H, Ridgway RA, Sansom OJ, Nathke IS. Spindle orientation bias in gut epithelial stem cell compartments is lost in precancerous tissue. Cell Stem Cell 2010, 6:175-181.

66. Schepers AG, Vries R, van den Born M, van de Wetering $\mathrm{M}$, Clevers H. Lgr5 intestinal stem cells have high telomerase activity and randomly segregate their chromosomes. EMBO J 2011, 30:1104-1109.

67. Snippert HJ, van der Flier LG, Sato T, van Es JH, van den Born M, Kroon-Veenboer C, Barker N, Klein AM, van Rheenen J, Simons BD, et al. Intestinal crypt homeostasis results from neutral competition between symmetrically dividing Lgr5 stem cells. Cell 2010, 143:134-144.

68. Lechler T, Fuchs E. Asymmetric cell divisions promote stratification and differentiation of mammalian skin. Nature 2005, 437:275-280.

69. Williams SE, Beronja S, Pasolli HA, Fuchs E. Asymmetric cell divisions promote Notch-dependent epidermal differentiation. Nature 2011, 470:353-358.

70. Poulson ND, Lechler T. Robust control of mitotic spindle orientation in the developing epidermis. J Cell Biol 2010, 191:915-922.

71. Clayton E, Doupe DP, Klein AM, Winton DJ, Simons $\mathrm{BD}$, Jones PH. A single type of progenitor cell maintains normal epidermis. Nature 2007, 446:185-189.

72. Charge SBP, Rudnicki MA. Cellular and molecular regulation of muscle regeneration. Physiol Rev 2004, 84:209-238.

73. Tatsumi R, Liu XS, Pulido A, Morales M, Sakata T, Dial S, Hattori A, Ikeuchi Y, Allen RE. Satellite cell activation in stretched skeletal muscle and the role of nitric oxide and hepatocyte growth factor. Am J Physiol Cell Physiol 2006, 290:C1487-C1494.

74. Fuchs E, Tumbar T, Guasch G. Socializing with the neighbors: stem cells and their niche. Cell 2004, 116:769-778.

75. Kuang SH, Kuroda K, Le Grand F, Rudnicki MA. Asymmetric self-renewal and commitment of satellite stem cells in muscle. Cell 2007, 129:999-1010.
76. Burkin DJ, Kaufman SJ. The alpha 7 beta 1 integrin in muscle development and disease. Cell Tissue Res 1999, 296:183-190.

77. Cornelison DDW, Wold BJ. Single-cell analysis of regulatory gene expression in quiescent and activated mouse skeletal muscle satellite cells. Dev Biol 1997, 191:270-283.

78. Shinin V, Gayraud-Morel B, Gomes D, Tajbakhsh S. Asymmetric division and cosegregation of template DNA strands in adult muscle satellite cells. Nat Cell Biol 2006, 8:677-687.

79. Conboy MJ, Karasov AO, Rando TA. High incidence of non-random template strand segregation and asymmetric fate determination in dividing stem cells and their progeny. Plos Biol 2007, 5:1120-1126.

80. Crittenden SL, Petcherski AG, Wickens MP, Kimble J. Distal tip cell control of germline stem cells in C-elegans. Dev Biol 2000, 222:224-224.

81. Crittenden SL, Troemel ER, Evans TC, Kimble J. Glp-1 is localized to the mitotic region of the C-elegans germ-line. Development 1994, 120:2901-2911.

82. Crittenden SL, Leonhard KA, Byrd DT, Kimble J. Cellular analyses of the mitotic region in the Caenorhabditis elegans adult germ line. Mol Biol Cell 2006, 17:3051-3061.

83. Siller KH, Doe CQ. Spindle orientation during asymmetric cell division. Nat Cell Biol 2009, 11:365-374.

84. Cabernard C, Doe CQ. Apical/basal spindle orientation is required for neuroblast homeostasis and neuronal differentiation in Drosophila. Dev Cell 2009, 17:134-141.

85. Suzuki A, Ohno S. The PAR-aPKC system: lessons in polarity. J Cell Sci 2006, 119:979-987.

86. Rusan NM, Peifer M. A role for a novel centrosome cycle in asymmetric cell division. J Cell Biol 2007, 177:13-20.

87. Rebollo E, Sampaio P, Januschke J, Llamazares S, Varmark H, Gonzalez C. Functionally unequal centrosomes drive spindle orientation in asymmetrically dividing Drosophila neural stem cells. Dev Cell 2007, 12:467-474.

88. Conduit PT, Raff JW. Cnn dynamics drive centrosome size asymmetry ito ensure daughter centriole retention in Drosophila neuroblasts. Curr Biol 2010, 20:2187-2192.

89. Januschke J, Llamazares S, Reina J, Gonzalez C. Drosophila neuroblasts retain the daughter centrosome. Nat Commun 2011, 2:243.

90. Megraw TL, Kao LR, Kaufman TC. Zygotic development without functional mitotic centrosomes. Curr Biol 2001, 11:116-120.

91. Basto R, Lau J, Vinogradova T, Gardiol A, Woods CG, Khodjakov A, Raff JW. Flies without centrioles. Cell 2006, 125:1375-1386. 
92. Basto R, Brunk K, Vinadogrova T, Peel N, Franz A, Khodjakov A, Raff JW. Centrosome amplification can initiate tumorigenesis in flies. Cell 2008, 133:1032-1042.

93. Farkas LM, Huttner WB. The cell biology of neural stem and progenitor cells and its significance for their proliferation versus differentiation during mammalian brain development. Curr Opin Cell Biol 2008, 20:707-715.

94. Wang X, Tsai JW, Imai JH, Lian WN, Vallee RB, Shi SH. Asymmetric centrosome inheritance maintains neural progenitors in the neocortex. Nature 2009, 461:947-955.

95. Bond J, Roberts E, Springell K, Lizarraga SB, Scott S, Higgins J, Hampshire DJ, Morrison EE, Leal GF, Silva EO, et al. A centrosomal mechanism involving CDK5RAP2 and CENPJ controls brain size. Nat Genet 2005, 37:353-355.

96. Bond J, Woods CG. Cytoskeletal genes regulating brain size. Curr Opin Cell Biol 2006, 18:95-101.

97. Cairns J. Mutation selection and natural-history of cancer. Nature 1975, 255:197-200.

98. Smith GH. Label-retaining epithelial cells in mouse mammary gland divide asymmetrically and retain their template DNA strands. Development 2005, 132:681-687.

99. Karpowicz P, Morshead C, Kam A, Jervis E, Ramunas J, Cheng V, van der Kooy D. Support for the immortal strand hypothesis: neural stem cells partition DNA asymmetrically in vitro. J Cell Biol 2005, 170:721-732.

100. Fei JF, Huttner WB. Nonselective sister chromatid segregation in mouse embryonic neocortical precursor cells. Cereb Cortex 2009, 19:I49-I54.
101. Karpowicz P, Pellikka M, Chea E, Godt D, Tepass U, van der Kooy D. The germline stem cells of Drosophila melanogaster partition DNA non-randomly. Eur J Cell Biol 2009, 88:397-408.

102. Sotiropoulou PA, Candi A, Blanpain C. The majority of multipotent epidermal. Stem Cells 2008, 26:2964-2973.

103. Waghmare SK, Bansal R, Lee J, Zhang YV, McDermitt DJ, Tumbar T. Quantitative proliferation dynamics and random chromosome segregation of hair follicle stem cells. Embo Journal 2008, 27:1309-1320.

104. Kiel MJ, He SH, Ashkenazi R, Gentry SN, Teta M, Kushner JA, Jackson TL, Morrison SJ. Haematopoietic stem cells do not asymmetrically segregate chromosomes or retain BrdU. Nature 2007, 449:238-242.

105. Yadlapalli S, Cheng J, Yamashita YM. Drosophila male germline stem cells do not asymmetrically segregate chromosome strands. J Cell Sci 2011, 124(pt 6):933-939.

106. Falconer E, Chavez EA, Henderson A, Poon SSS, McKinney S, Brown L, Huntsman DG, Lansdorp PM. Identification of sister chromatids by DNA template strand sequences. Nature 2010, 463:93-97.

107. Tajbakhsh S, Gonzalez C. Biased segregation of DNA and centrosomes - moving together or drifting apart? Nat Rev Mol Cell Biol 2009, 10:804-810.

108. Garcia-Mata R, Gao YS, Sztul E. Hassles with taking out the garbage: aggravating aggresomes. Traffic 2002, 3:388-396.

109. Rujano MA, Bosveld F, Salomons FA, Dijk F, van Waarde MAWH, van der Want JJL, de Vos RAI, Brunt ER, Sibon OCM, Kampinga HH. Polarised asymmetric inheritance of accumulated protein damage in higher eukaryotes. Plos Biol 2006, 4:2325-2335.

\section{FURTHER READING}

Xie T. Germline stem cell niches. In: Lin H, Donahoe P, (eds.). StemBook: The Stem Cell Research Community. Cambridge, MA: Harvard Stem Cell Institute. StemBook; 2008. doi:10.3824/stembook.1.23.1. Available at: http://www.stembook.org.

Silva H, Conboy IM. Aging and stem cell renewal. In: Gilliland G (ed.). StemBook. The Stem Cell Research Community. Cambridge, MA: Harvard Stem Cell Institute. StemBook; 2008. doi:10.3824/stembook.1.11.1. Available at: http://www. stembook.org. 